\title{
Recalce en condiciones de emergencia: 40 viviendas cercanas al río Guadalquivir, España
}

\author{
Foundation recovery under emergency conditions. 40 flats \\ by the Guadalquivir river, Spain
}

A. Ferreira Sánchez ${ }^{(*)}$, M. a D. Alba Rodríguez $^{(*)}$, A. Ramírez-de-Arellano ${ }^{(*)}$, M. Marrero $^{(*)}$

\section{RESUMEN}

Las intervenciones en edificaciones en circunstancias de emergencia, y en concreto los recalces de cimentaciones son circunstancias poco documentadas dentro de la rehabilitación. Esto se debe, en parte, a la complejidad técnica y la tendencia a evitar hacer públicos datos que puedan conllevar responsabilidades derivadas.

En el artículo se expone el recalce de un edificio de 40 viviendas en Sevilla, cercanas al Río Guadalquivir, afectado por la construcción contigua de un aparcamiento subterráneo. También se establece una comparativa entre tres técnicas consideradas, y finalmente, se explica, técnica y económicamente el método de micropilotes adoptado. Éste se elige, entre otras razones, debido a la calidad del suelo. De la experiencia se ha podido inferir que el éxito de la intervención se ha conseguido debido a tres factores: la organización de la obra, el estudio de los plazos y la implicación de los propietarios afectados.

Palabras clave: Recalce; cimentación; emergencia; organización; planificación; micropilotes; rehabilitación.

\section{ABSTRACT}

The reconstruction works in buildings under emergency conditions and, more specifically, the foundation repaired has been poorly documented in rehabilitation. This is due, in some extend, to not make public this type of reports mainly due to its complexity and possible related responsibilities.

In the present article, the foundation recovery of a building with 40 flats, located in Seville, Spain, near the Guadalquivir River which was affected by the excavations of an underground parking lot are explained. Three different techniques are considered and compared; finally, the micropile solution implemented is explained, technically and economically. Micropiles were chosen due to, among other aspects, the soil quality. The experience gives rise to three main factors in a successful work: the organization, the scheduling and the neighbors' participation in the work programming.

Keywords: Foundation recovery; foundation; emergency; organization; planification; micropile; rehabilitation

${ }^{(*)}$ Universidad de Sevilla (España).

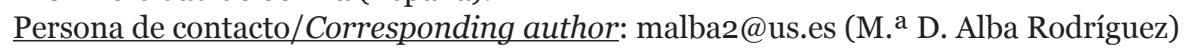

Cómo citar este artículo/Citation: Ferreira Sánchez, A., Alba Rodríguez, M. ${ }^{a}$ D., Ramírez-de-Arellano, A., Marrero, M. (2016). Recalce en condiciones de emergencia: 40 viviendas cercanas al río Guadalquivir, España. Informes de la Construcción, 68(541): e133, doi: http://dx.doi.org/10.3989/ic.14.132.

Licencia / License: Salvo indicación contraria, todos los contenidos de la edición electrónica de Informes de la Construcción se distribuyen bajo una licencia de uso y distribución Creative Commons Reconocimiento no Comercial 3.o. España (cc-by-nc). 


\section{INTRODUCCIÓN}

La recuperación de edificios es un tema ampliamente estudiado en el ámbito de la construcción, si bien algunas parcelas singulares, como las intervenciones de emergencia, no están lo suficientemente desarrolladas. En países con potenciales riesgos que generen situaciones de emergencia en los edificios, como terremotos, se han desarrollado protocolos de evaluación (1) o procesos para la toma de decisiones en la posterior rehabilitación (2). Si bien en España, casos como el terremoto de Lorca en 2011 o los daños causados por la construcción del metro en el barrio El Carmel en 2005, que generaron situaciones de emergencia, no han sido estudiados suficientemente.

En concreto, los recalces de cimentaciones afectadas por asentamientos, por ser el elemento más perjudicado y cuya reparación es crítica para restablecer la interacción terrenoestructura, son los procesos más interesantes de estudiar en este sentido (3) (4) (5) (6). En el ámbito de los recalces es necesario estudiar técnicas, procesos, soluciones y resultados de casos reales, creando referencias que permitan avanzar en su estudio (7). Aspectos como la intervención en el terreno (8), los costes derivados o la gestión de la recuperación de edificios en circunstancias de emergencia social, tampoco han sido profusamente investigados.
La escasa divulgación técnica relativa a este tipo de actuaciones, puede ser debida a la tradicional reticencia a publicar datos técnicos susceptibles de responsabilidades. Esto lleva a que, en general, los técnicos que deban enfrentarse a este tipo de situaciones, no tengan referencias previas en las que poder basar sus decisiones y que, en esos escenarios de incertidumbre, se opte por soluciones conservadoras y sobredimensionadas, tanto a nivel técnico como económico.

En el presente artículo, la Universidad de Sevilla pone a disposición de la comunidad la experiencia adquirida durante las obras de recalce de 40 viviendas en la «Barriada RENFE» cuya cimentación fue afectada por la excavación colindante de la nueva Facultad de Ciencias de la Salud.

La denominada «Barriada RENFE» se encuentra situada al norte del casco histórico de Sevilla, en el popular barrio de La Macarena. El origen de este conjunto de edificios se enmarca en la mitad del S.XX, cuando debido a la necesidad de dotar a los trabajadores de RENFE de viviendas, esta institución conjuntamente al Instituto Nacional de la Vivienda promovió su ejecución en los terrenos aledaños a sus instalaciones ferroviarias, terrenos de marcado carácter aluvial debido a su cercanía al río Guadalquivir y que hasta los años 80 fue un tramo vivo.

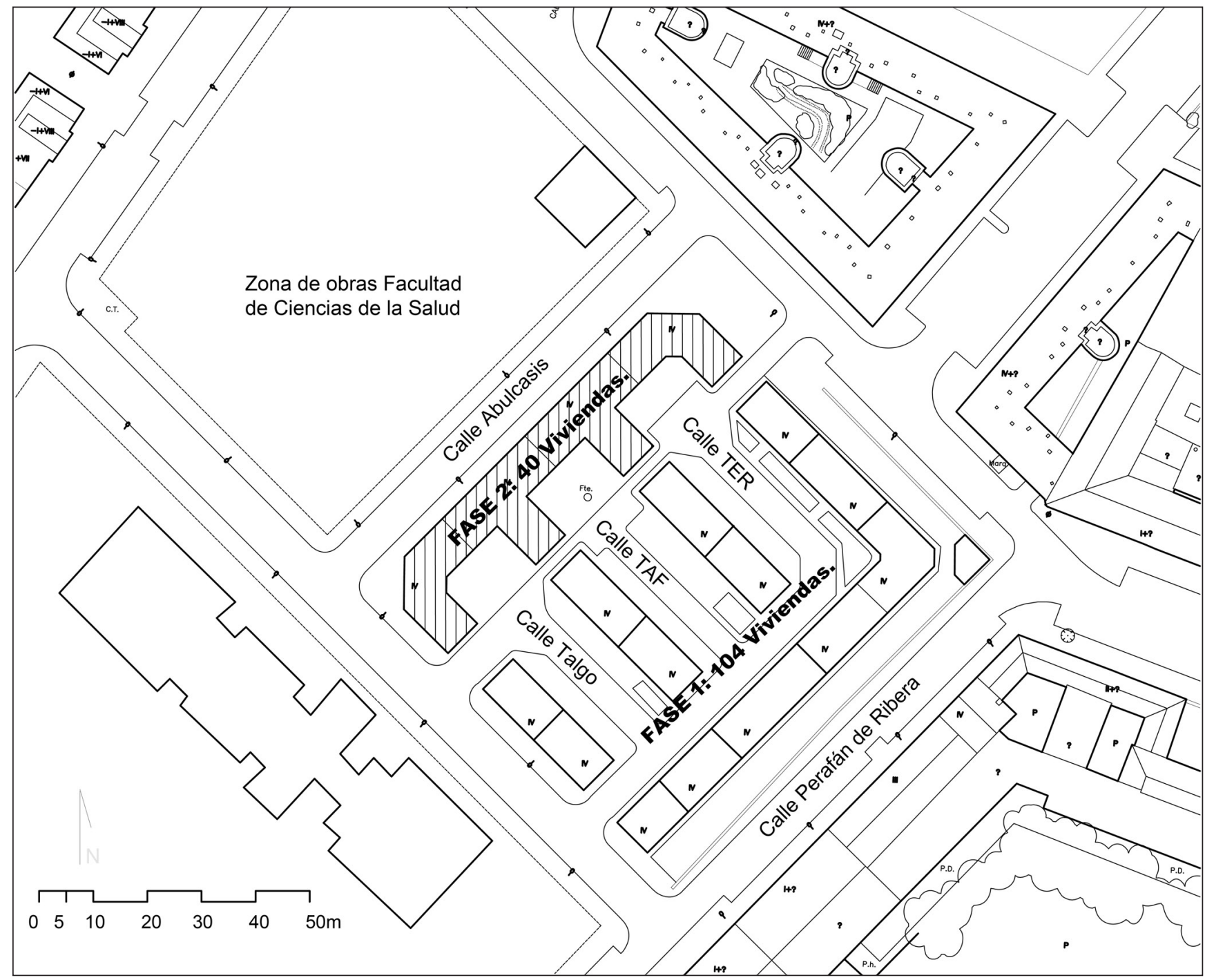

Figura 1. Entorno de la denominada «Barriada RENFE» (Sevilla). 
Su ejecución se realizó en dos fases, la primera de 104 viviendas y la segunda de 40, siendo dirigidas ambas por el arquitecto D. Fernando Barquín entre 1950 y 1960. La dilatación del plazo de las obras fue debido a circunstancias económicas adversas para la inicial empresa adjudicataria por causa de aspectos técnicos de las obras según se pudo constatar en el expediente administrativo de las obras y proyecto original (9).

El conjunto (Figura 1) se caracteriza por ser un grupo de bloques cerrados que se desarrollan en $\mathrm{PB}+3$ y que mantienen criterios similares en estética y distribución, siguiéndose en su diseño los criterios que establecía el Instituto Nacional de la Vivienda en la época para la realización de viviendas de carácter social.

Constructivamente ambas fases difieren esencialmente en el tipo de cimentación, mientras la primera se cimentó sobre pilotes de 420 y $630 \mathrm{~mm}$ de diámetro y 20 metros de profundidad, la segunda por motivos económicos se realizó sobre zapatas corridas (9). El resto de los componentes no difiere en grandes rasgos, realizándose su estructura con fábricas de ladrillo y forjados realizados in situ.

El conjunto residencial no ha sufrido variaciones relevantes a lo largo de los años, hasta la ejecución, en una parcela colindante, de las obras de la nueva Facultad de Ciencias de la Salud de la Universidad de Sevilla. Tras finalizar el proceso de excavación del vaso de este edificio colindante, se retiró la rampa de acceso de tierra que se había dejado en la excavación y causó el desplazamiento del muro pantalla, longitudinal a la calle Abulcasis (ver Figura 1), al no estar bien anclado según se constató por el desplazamiento sin fracturas en el lienzo o anclajes. En este momento, empezaron a detectarse la aparición de daños en el edificio de 40 viviendas, con la aparición de grietas y fisuras en muros y tabiquería.

El desplazamiento produjo un proceso de descompresión del terreno que afectó a la cimentación superficial del edificio y se tradujo en un asiento diferencial. La gravedad y desarrollo de la patología requirió la intervención de los Servicios de Emergencia del Ayuntamiento de Sevilla y el desalojo de las viviendas. Durante varios días, hasta la estabilización del muro pantalla mediante el vertido de una berma de bolos, la progresión de los daños fue en aumento hasta dañar gravemente la cimentación, los muros de carga y la tabiquería interior, además de producir un asentamiento del terreno en el entorno.

La crisis social derivada para las 40 familias desalojadas y la relación causa-efecto del proceso hizo que la Universidad se implicase activamente y adoptara una actitud de responsabilidad social, haciéndose cargo de la reparación del edificio, y la asistencia social a los afectados. En el presente artículo, en primer lugar, se expone el análisis de los daños, diagnóstico, posibles soluciones y la finalmente adoptada. Se evalúa la solución tanto técnica como económicamente.

\section{ANÁLISIS DE LOS DAÑOS}

La patología afectó a la totalidad de la edificación, si bien la magnitud de los daños variaría en función de la proximidad al punto de mayor asentamiento. La estructura de fábrica de ladrillo se vio afectada generando arcos de descargas longitudinales y transversales de diversa magnitud. El edificio quedó dañado estructuralmente y perdió la funcionalidad de todos sus espacios.

Ante la necesidad de definir el proceso de recuperación se estableció la siguiente metodología de análisis: caracterización geométrica y material de los elementos estructurales, caracterización del terreno en el entorno del edifico afectado y documentación gráfica de las patologías. La caracterización del terreno, se basó en la experiencia previa del equipo técnico encargado y asesorados por una reputada empresa de control y ensayos, se compuso de los siguientes ensayos:

- sondeos con extracción de testigos hasta una profundidad media de 33 metros.

- 6 ensayos presiométricos.

- 45 ensayos SPT de penetración estándar en sondeos (10).

- 3 tubos piezométricos para el control del nivel freático.

- 1 ensayo de agresividad a los hormigones del agua freática.

- Ensayos de granulometría, límites de Atterberg, peso específico, humedad natural, compresión simple y corte directo en laboratorio de 33 muestras inalteradas y 3 testigos parafinados.

- Seguimiento del desarrollo de los daños mediante la instrumentación del edificio, compuesta de nivelación digital milimétrica y puntos de seguimiento en cubierta para estaciones totales.

Tras la finalización de los ensayos necesarios para la determinación de las características geotécnicas del terreno y sus estratos (Figura 2), se consideró necesario un reconocimiento geofísico por la presencia de agua y el sonido de escorrentías en el trasdós del muro pantalla. Este tipo de pruebas, propias de la ingeniería del terreno en obra civil, son raramente aplicadas en el ámbito de la edificación, si bien la necesidad de conocer en profundidad las características del terreno y los indicios de la posible existencia de oquedades, recomendaron su aplicación en este caso. La técnica se basa en la interpretación de los datos y su gestión mediante un software específico que muestran un corte vertical de terreno en la longitud sometida a reconocimiento. Los datos provienen de la caracterización de la resistividad eléctrica del terreno, teniendo en cuenta que mayor presencia de porosidad supone mayor resistividad eléctrica. Esto permite obtener un perfil del terreno que mediante variación de colores representa la consistencia del terreno.

\section{Características geotécnicas}

La información obtenida en los ensayos determinó que el terreno presentaba deficientes características portantes, heterogeneidades en sus estratos y se detectaron oquedades con indicios de corrientes subterráneas. Siendo un terreno propio de zonas aluviales con materiales de escasa consistencia, los Ensayos de Penetración Estándar (SPT) realizados en cada uno de los 6 sondeos, mostraron que el terreno presentaba escasa resistencia por encima de 20 metros de profundidad. Asimismo, se observó que el nivel freático presentaba grandes variaciones en la zona cercana a la pantalla, lo que reforzó la teoría de la presencia de corrientes subterráneas.

Del reconocimiento geofísico del terreno se pudo extraer, mediante la interpretación de los gráficos, la presencia de zonas de elevada resistividad propias del terreno descomprimido, y franjas de terreno altamente conductivo entre 4 y 11 metros de profundidad. Se presentaron discontinuidades verticales 


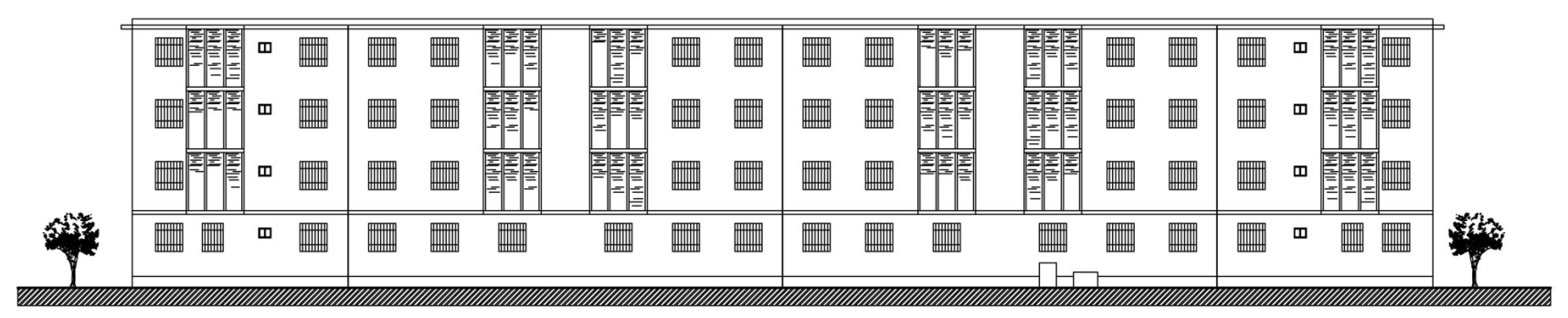

ALZADO PRINCIPAL

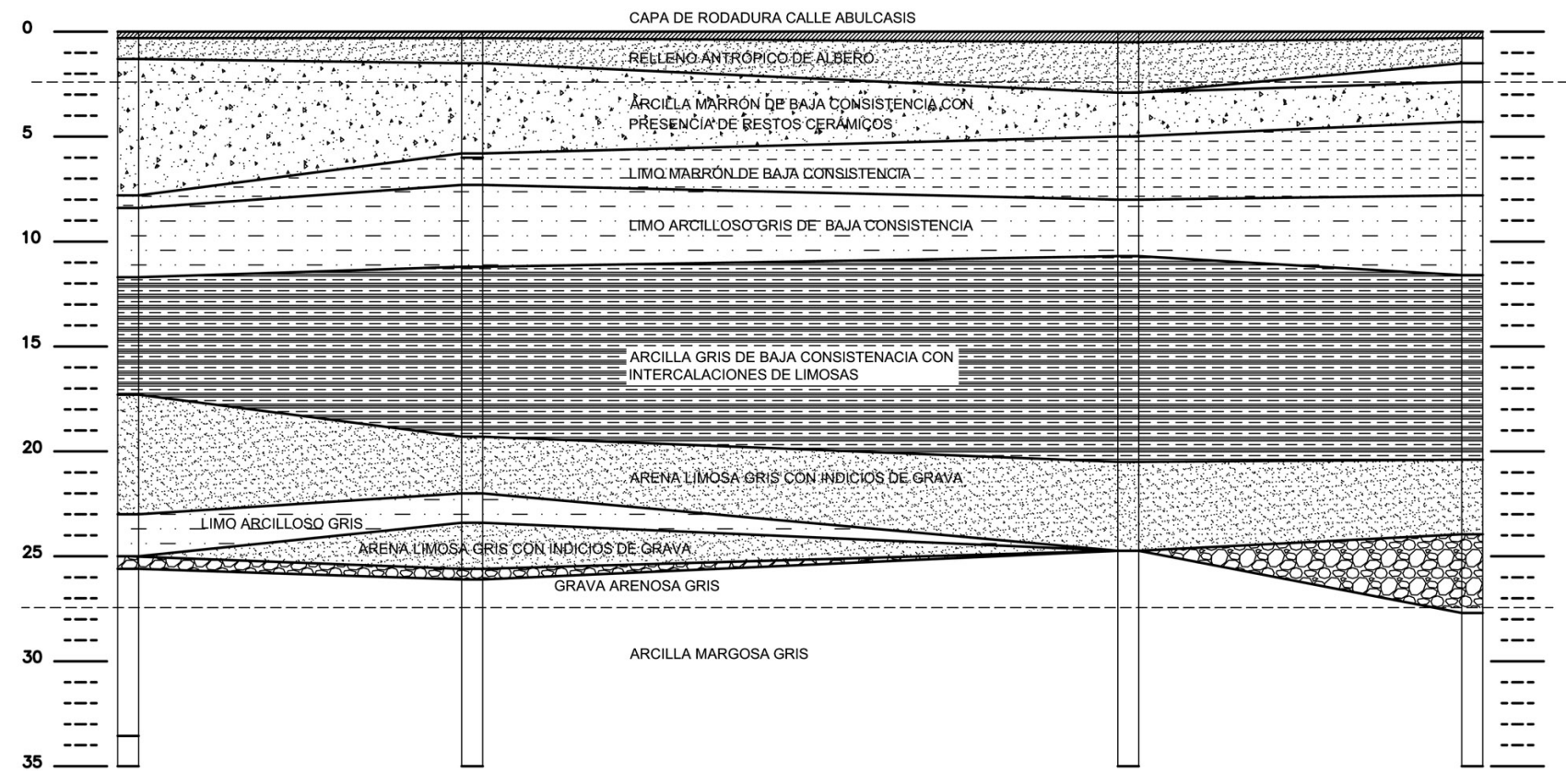

Figura 2. Estratificación y caracterización del terreno.

que mostraban las fracturas del terreno producidas por el desplazamiento horizontal.

Junto al reconocimiento del terreno y la monitorización del edificio se realizó una caracterización de los daños en el edificio afectado que, siguiendo la escala de Burland (11), se calificaron de graves a moderados, con presencia de hundimientos en las soleras, agrietamientos en las fábricas y descuelgues de tramos de muro de carga.

\section{DIAGNÓSTICO}

El análisis de los datos recopilados en la campaña de reconocimiento permitió extraer las siguientes conclusiones:

- La seguridad estructural del edificio estaba comprometida por los daños cuyo origen estaban en el desplazamiento del muro pantalla, con el consiguiente proceso de descompresión y desplazamiento del terreno.

- El terreno mostró tras los ensayos geotécnicos malas características portantes hasta los 20 metros bajo rasante, aunque el perfil litológico se corresponde con el típico de la zona (limos y arcillas de baja consistencia), y aparecen fuertes heterogeneidades en sus estratos.

- El desarrollo de la patología, a pesar de estar controlado, continuaba en progreso. Siendo necesaria la monitorización durante el proceso de reparación.

- La cimentación, afectada por los asientos diferenciales, requiere de la intervención para asegurar su estabilidad.
- Cualquier solución a adoptar debe permitir finalizar las obras, que concluyen con la recepción de las viviendas por sus propietarios, en el plazo de un año desde el accidente.

Finalizado este proceso, se inició el proceso de ejecución de las obras de reparación del edificio y se continuó con los trabajos de construcción de la nueva Facultad de Ciencias de la Salud, y, para evitar las posibles interferencias entre obras, se establecieron procesos de coordinación entre los responsables de ambas, desarrollándose la planificación conjunta de los trabajos y la organización de los espacios comunes.

\section{SOLUCIONES DISPONIBLES}

El desarrollo técnico de la edificación a partir de la segunda mitad del S. Xx, ha permitido disponer de una amplia variedad de técnicas destinadas a recuperar la interacción entre terreno y cimentación. La multitud de variantes, de materiales a utilizar y la capacidad de control de los procesos, han llevado a poder resolver todas las casuísticas posibles dando una respuesta concreta a cada caso.

Se propone como clasificación para los recalces tres grandes bloques en los que aglutinar las técnicas aplicables:

- Técnicas tradicionales.

Consistentes en la profundización de la cota de apoyo de la cimentación hasta un estrato más competente o la am- 


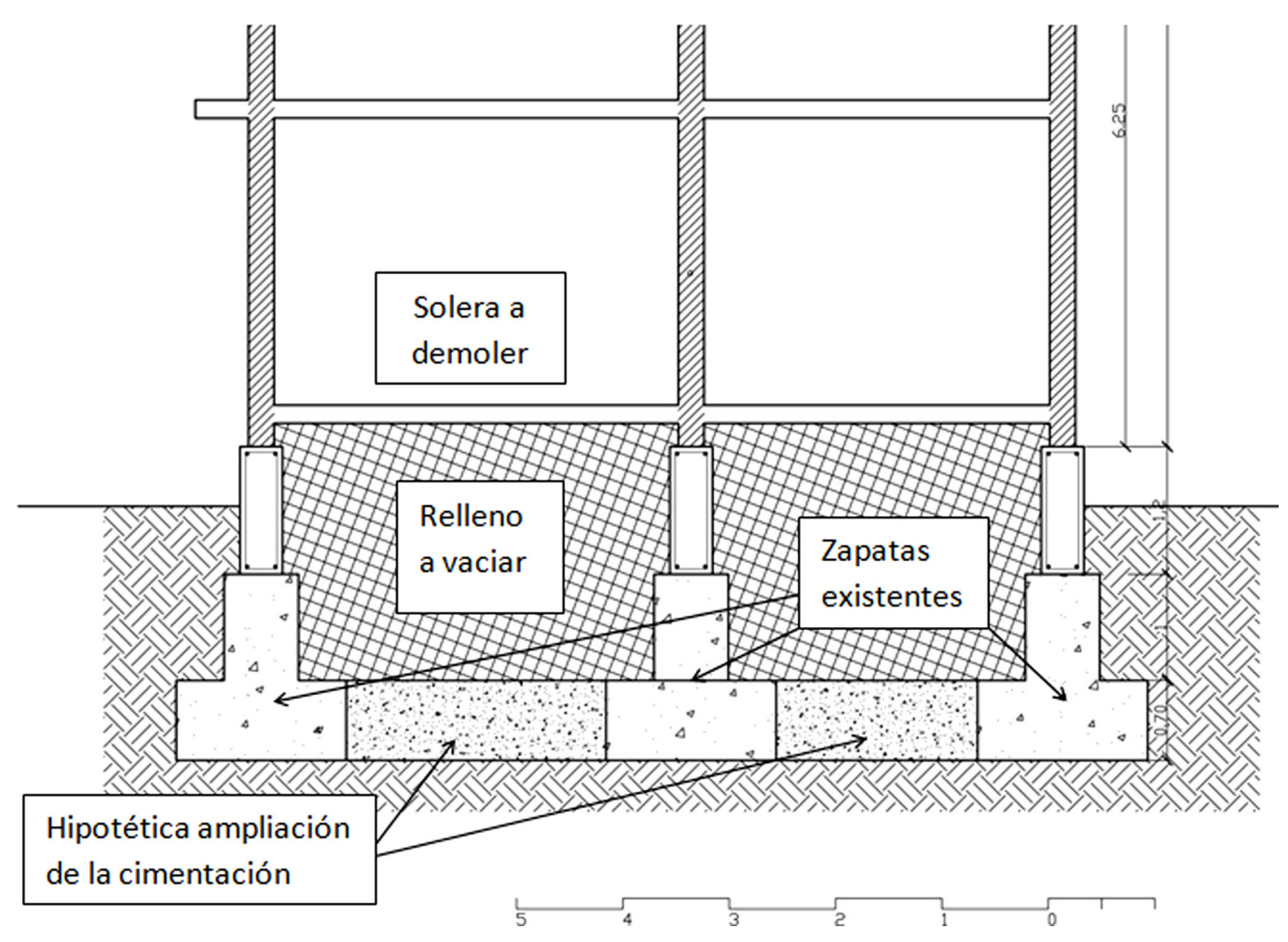

Figura 3. Sección transversal. Esquema de hipotético recalce por aumento de superficie.

pliación de la superficie de apoyo para reducir los esfuerzos transmitidos al terreno.

- Intervención en el terreno subyacente a la cimentación. Realizados por medio de inyecciones de diversos materiales directamente bajo la cimentaciones y con la finalidad de mejorar las características de este terreno. A este grupo corresponden las inyecciones armadas de cemento y las inyecciones de resinas expansivas de poliuretano (ERP).

- Recalce por micropilotes.

Buscan la transmisión directa de las cargas de la cimentación a un estrato competente por medio de elementos estructurales. Este grupo se compone básicamente de los micropilotes con cada una de sus variantes.

\subsection{Técnicas tradicionales}

Las técnicas tradicionales de recalce, consisten en la profundización de la cota de apoyo o la ampliación de su superficie. Descartada la posibilidad técnica de aumentar la profundidad del apoyo hasta 20 metros bajo rasante, la segunda opción requiere la realización del vaciado completo del interior del edificio hasta la cota de apoyo de la cimentación (Figura 3). Ello conlleva el uso de maquinaria de excavación de escasas dimensiones y de bajo rendimiento, con un aumento de los costes y los plazos de ejecución.

Por otro lado, en los procesos de asentamiento diferencial, la no intervención sobre el terreno descomprimido puede dar lugar a asentamientos diferidos en el tiempo. En el presente estudio, a pesar de controlar el origen del desplazamiento, no se podría descartar este hecho por lo que el uso de técnicas tradicionales no daría respuesta a las necesidades y fueron descartadas.

\subsection{Intervención en el terreno subyacente a la cimentación}

La intervención en el terreno subyacente a la cimentación presenta dos variantes principales que interesa diferenciar, dada la diferencia en sus sistemas de intervención y en el material a utilizar: inyección de cemento armado o de resinas expansivas.

\subsubsection{Inyecciones de cemento armadas}

La multitud de tipos de inyecciones de cemento que da respuesta a las demandas de distintos terrenos o necesidades (5) son desconocidas en el ámbito de la edificación, a pesar de dar excelentes respuestas frente a necesidades complejas. El elevado control, tanto de la presión como del volumen de material inyectado, que se puede aplicar sobre esta técnica la hace muy adecuada para construcciones de sótanos (12); e ir más allá de la intervención sobre el terreno y realizar refuerzos de estructuras en el patrimonio edificado (13).

Esta técnica está basada en la perforación del terreno hasta la zona subyacente a la cimentación, que tras la introducción de un abanico de tuberías de acero dotadas de válvulas anti retorno, realiza inyecciones de lechada de cemento destinadas a mejorar las características del suelo (Figura 4). Esta mejora se produce tras la rotura hidráulica del terreno y el fraguado de la red de lechada inyectada. La dosificación y características de la lechada de cemento varían en función de la viscosidad, la resistencia y la velocidad de fraguado que se pretenda conseguir. Otros factores a tener en cuenta son la agresividad del terreno o presencia de cloruros y la presión de la inyección. 
Este tipo de inyecciones realizadas por medio de tubos manguito, permiten que se realicen tanto de manera unitaria, en la totalidad de la longitud del tubo, como de manera selectiva por cada una de las válvulas. Con el uso de obturadores se pueden realizar incluso de manera secuencial, repitiendo la inyección en puntos concretos si se considera necesario. A pesar de lo que pueda parecer, estos sistemas permiten que se establezca un elevado control sobre las inyecciones, donde con la supervisión de las cantidades de material, las presiones de inyección y las densidades de éste, unidas a un buen conocimiento del terreno se puede valorar la efectividad de la intervención bajo rasante.

El control material se complementa con la nivelación milimétrica del edificio para así detectar durante el proceso posibles levantamientos en el edificio fruto de la presión de inyección. Asimismo, los tubos de acero para la inyección colaboran en la resistencia del tratamiento, actuando como armaduras en el terreno tratado. Por último, como únicamente se actúa sobre el terreno, no es necesario confiar la funcionalidad de la solución a enlaces entre la cimentación existente y el sistema de recalce.
En la obra objeto de estudio, la aplicación de esta solución para la mejora del terreno subyacente hubiese sido de fácil aplicación. No siendo necesaria la ejecución de ninguna medida auxiliar para la realización del recalce en el interior del edificio, puesto que desde el exterior y con una geometría adecuada se puede llegar a actuar incluso bajo la cimentación del muro intermedio del edificio siniestrado.

Dadas las características aluviales del terreno y con elevado nivel freático, se necesitarían realizar perforaciones de alivio para evitar subpresiones. La aplicación de esta técnica supone el aumento de la presión intersticial del terreno, que se refleja en una emanación del agua contenida en el terreno.

La técnica de inyección armada en el caso objeto de estudio hubiese presentado las siguientes ventajas y desventajas:

- Es un punto a favor el hecho de que las inyecciones se puedan realizar desde el exterior del edificio, no siendo necesario realizar demoliciones en el interior y minorando los plazos de ejecución.

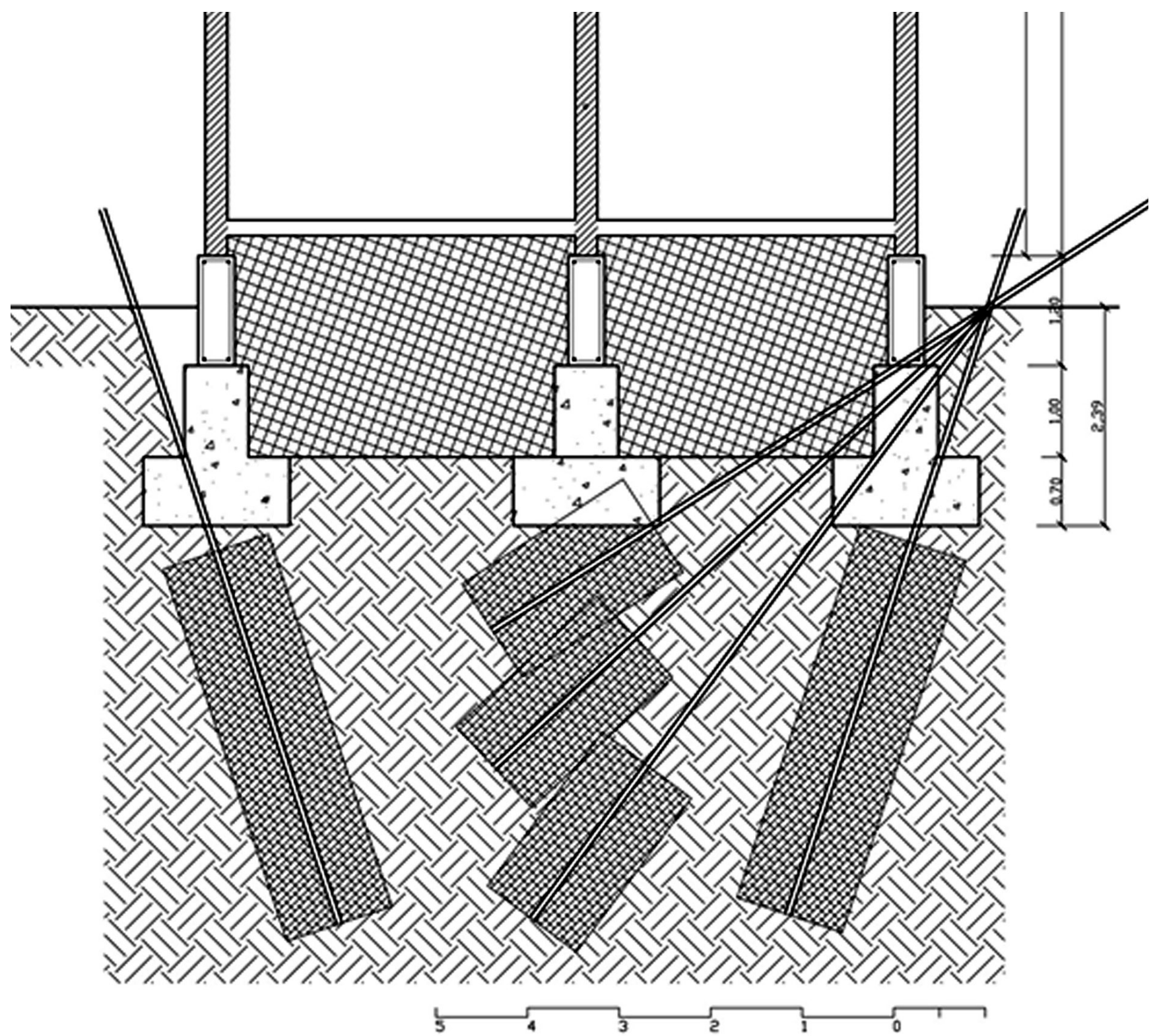

Figura 4. Detalle de la posible solución por inyecciones armadas en la obra. 
- Conociendo a posteriori la problemática para la perforación de las zapatas aplicada a los micropilotajes, el hecho de que esta técnica no requiera intervenir sobre la cimentación de manera directa hubiera presentado ventajas.

- Basándonos en las experiencias descritas en publicaciones científicas (5), la solución hubiese podido ser económicamente competitiva.

- La aplicación de esta técnica se habría limitado a la franja de terreno afectada por los bulbos de presión de las zapatas (5 metros de profundidad bajo la cimentación). La combinación de factores como: la baja consistencia de los estratos hasta 20 metros de profundidad, la presencia de escorrentías subterráneas y que el fondo de la excavación se sitúa 12 metros por debajo de la cota de apoyo, hubiera dejado una franja de terreno de 8 metros de profundidad sin tratar y susceptible aún de movimientos durante las obras o asentamientos diferidos.

\subsubsection{Inyecciones de Resinas Expansivas de Poliuretano (EPR)}

Este sistema de intervención comparte con el anterior el hecho de actuar exclusivamente sobre el terreno, su origen también se relaciona con el de las inyecciones de cemento, si bien en un punto del desarrollo técnico se decidió sustituir la inyección el cemento por otro material más activo. Las aplicaciones de este sistema van más allá de los recalces, también se usan para: la protección frente al sismo, las vibraciones de otro origen y los efectos de suelos expansivos. Las inyecciones se realizan con un poliuretano compuesto por la mezcla de un isocianato y un poliol en proporciones variables en función del tipo de poliuretano. Estos materiales al mezclarse generan una reacción exotérmica de polimerización que desprende $\mathrm{CO}_{2}$, que queda ocluido en el interior de la masa generando células cerradas que se expanden hasta finalizar la reacción. La reacción no dura más allá de 6-10 minutos, alcanzándose en una hora resistencias próximas a la total y en 24 h la máxima posible (14).

El sistema consiste en realizar una serie de perforaciones de escaso diámetro (hasta $\varnothing 26 \mathrm{~mm}$ ) bajo la longitud de la cimentación (a distancias entre 0,5 y 1,5 m) y colocar varillas por las que realizar la inyección de la mezcla (Figura 5). La inyección se realiza a baja o muy baja presión y en distintos puntos bajo la cimentación con la finalidad de realizar columnas de terreno mejorado tras la expansión de la resina que aumente su resistencia.

La expansión volumétrica de estas resinas en un entorno sin presión supera en 30 veces su volumen inicial; existen datos en investigaciones realizadas sobre estos materiales en los que se establece que resistencia puede alcanzar en su expansión entre 0,20 y 10,00 MPa para densidades de 0,5$10,00 \mathrm{kN} / \mathrm{m}^{3}(15)$.
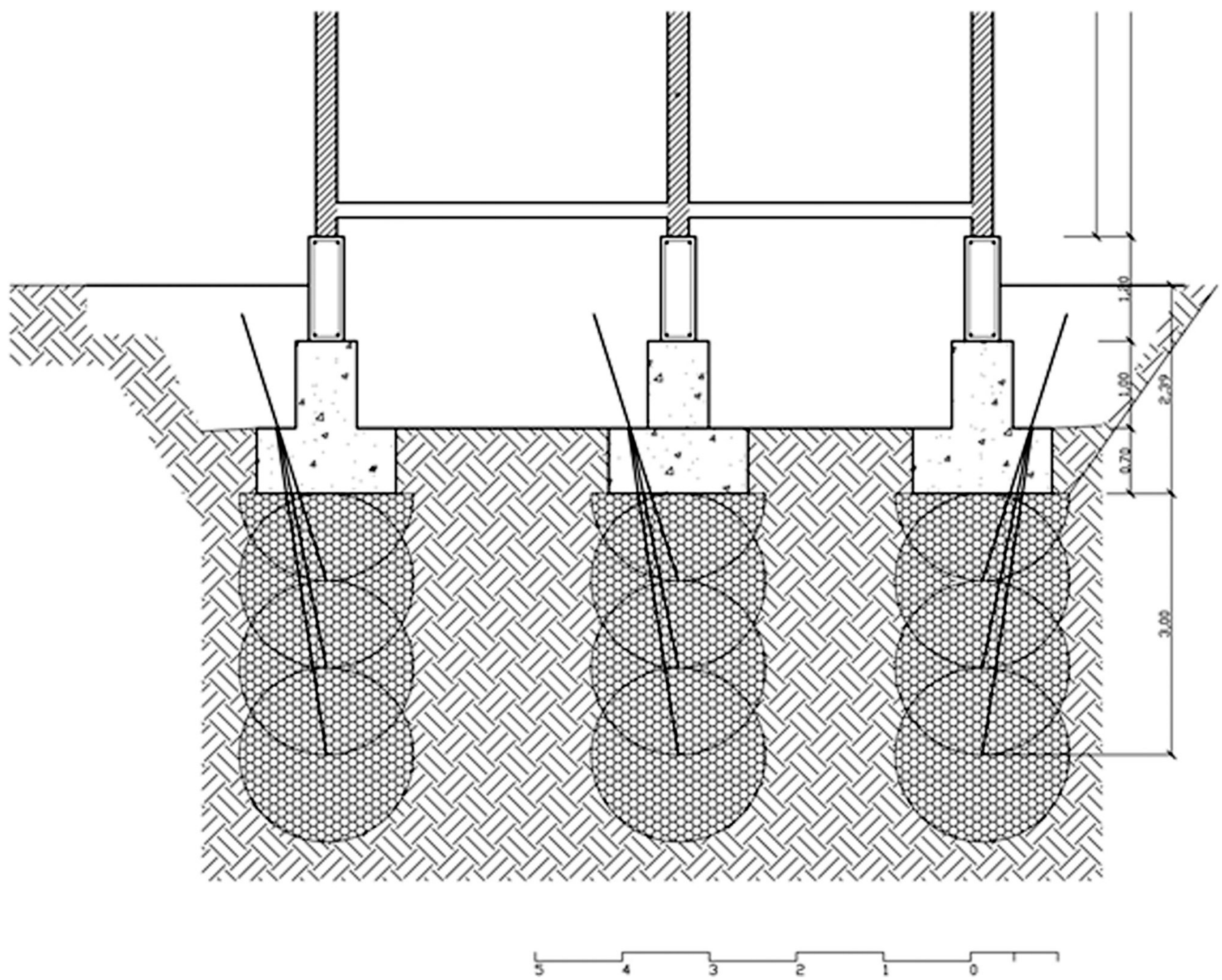

Figura 5. Esquema de la aplicación de inyecciones de resina expansiva en la obra. 
Hay que indicar que en esta técnica no se puede controlar la presión que se genera en el terreno puesto que depende de la expansividad de la resina, por ello se hace necesario realizar nivelaciones de control milimétrico, que permitan ir observando la evolución de las inyecciones, su efectividad y poder realizar reinyecciones en los puntos necesarios.

La rápida reacción de la resina impide que esta se disperse más allá de unos 2 metros desde el punto de inyección, lo que permite focalizar las acciones a puntos concretos y evitar pérdidas innecesarias de material. Tras la reacción de la resina y su aumento de volumen, queda un poliuretano totalmente inerte que no reacciona con ninguno de los materiales que se pueden encontrar bajo una cimentación, aunque en ninguno de los estudios consultados se ha podido encontrar referencias a la durabilidad de este material (16).

La aplicación de esta técnica requiere los siguientes sucesos:

- Realizar el estudio del terreno.

- Establecer una referencia de nivelación en su estado inicial.

- Realizar perforaciones para la colocación de los tubos de inyección en los puntos y alturas a inyectar.

- Realizar las inyecciones a la vez que se controla la nivelación y la efectividad de estas, ya sea de estabilización del terreno como de recuperación de los asentamientos.

La inyección se realiza en dos fases, una primera de pre-consolidación justo debajo del plano de apoyo de la cimentación y otra posterior en los puntos definidos al efecto. La técnica permite realizar varias inyecciones secuenciales en el mismo punto en caso de que con la primera inyección no se haya conseguido obtener los resultados deseados.

En cualquier caso, como en las soluciones descritas previamente habría que valorar los siguientes factores:

- Las inyecciones secuenciales no son homogéneas, crean discontinuidades en el poliuretano y que los resultados de la resistencia adquirida están un $62 \%$ por debajo de los resultados obtenidos en los laboratorios (16). Lo que dista bastante de los resultados obtenidos de los ensayos realizados en la Universidad de Padua bajo los criterios de la norma UNI 6350-68 Materias plásticas celulares rígidas-Determinación de las características a la compresión (17).

- La aplicación de esta técnica en terrenos de tipo aluvial y de grano fino no son excesivamente recomendables, ya que se crean sobrepresiones que pueden producir ligeros asientos diferidos (18).

- Existen referencias bibliográficas que indican que la solución no es adecuada para el tipo de terreno sobre el que se asienta el edificio del caso de estudio (18).

- Una ventaja es el elevado rendimiento y el escaso consumo de material para la realización del recalce.

- Hubiera sido necesario realizar grandes movimientos de terreno en el interior del edificio para acceder a las zapatas intermedias, reduciendo los buenos rendimientos de la aplicación.

- La aplicación de este sistema requiere que el terreno fuese comprimible en las inyecciones. La presencia de una zona descomprimida por el desplazamiento del muro pantalla y la necesidad de realizar el recalce a la vez que la consolidación, invalida su viabilidad.

\section{SOLUCIÓN ADOPTADA}

\subsection{Recalce con micropilotes}

Para trasladar las cargas del edificio desde la cimentación al único estrato competente situado en las margas azules a $25 \mathrm{~m}$ de profundidad media, se decidió acometer el recalce de la cimentación con 232 micropilotes de tipo IR, de $27 \mathrm{~m}$ de longitud y $140 \mathrm{~mm}$ de diámetro de perforación, con camisa de acero tipo N8o con $60,3 \mathrm{~mm}$ de diámetro y entre 5 y 10 grados de inclinación respecto del eje vertical; la distribución de estos fue al tresbolillo y a lo largo de todas las zapatas, separados $2 \mathrm{~m}$ entre ejes de micropilotes en zapatas exteriores y $1 \mathrm{~m}$ en interiores.

Se selecciona el micropilote IR (Figura 6), para conseguir un bulbo en la parte inferior de las zapatas que favoreciese el apoyo del recalce en la zona de terreno de peor calidad, mediante la inyección a presión de lechada de cemento a través de las válvulas antiretorno.

Durante el desarrollo de las obras se produjeron una serie de contratiempos que comprometieron la planificación y lleva-

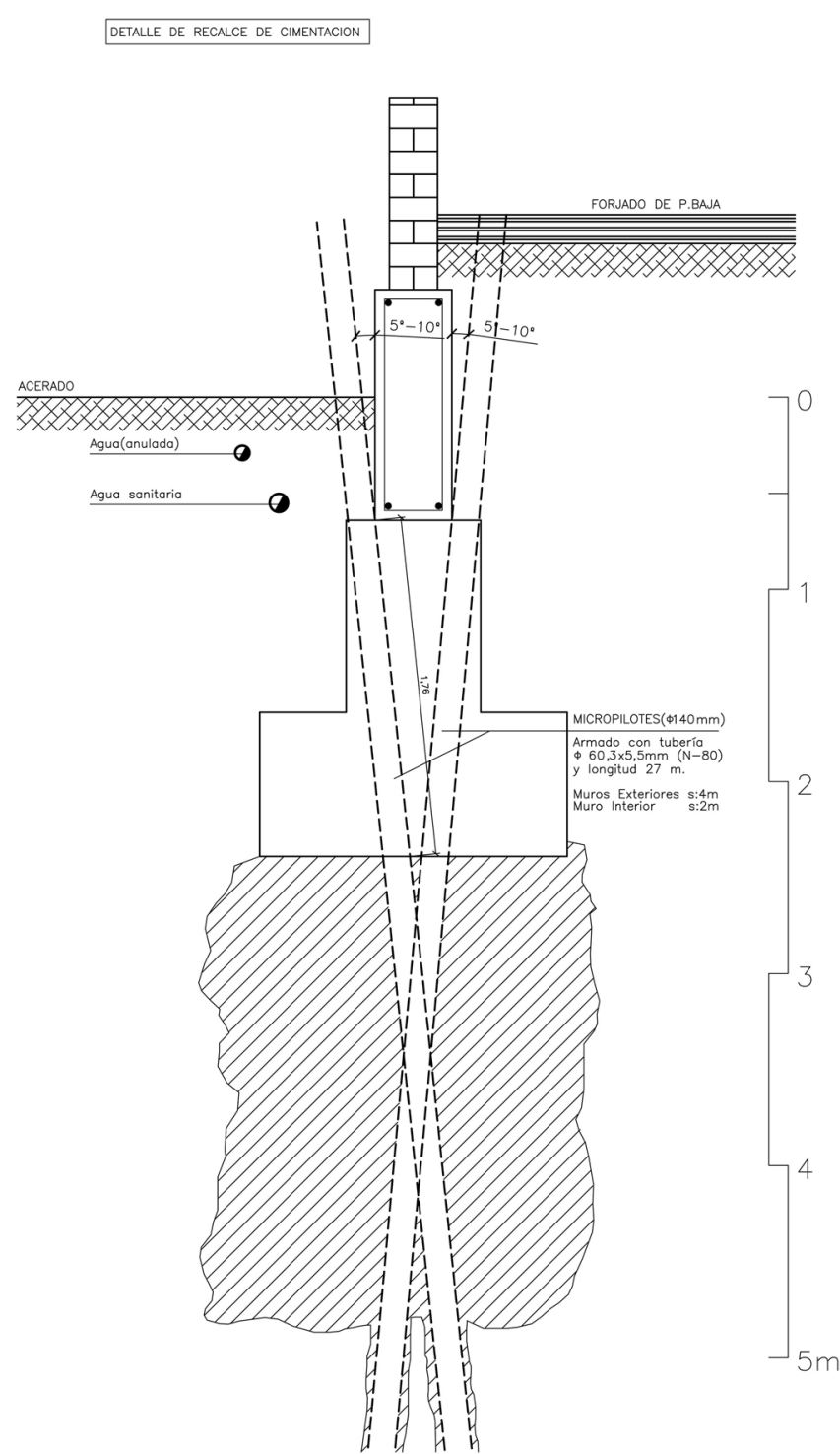

Figura 6. Detalle del recalce de la cimentación. Fuente: Universidad de Sevilla. 

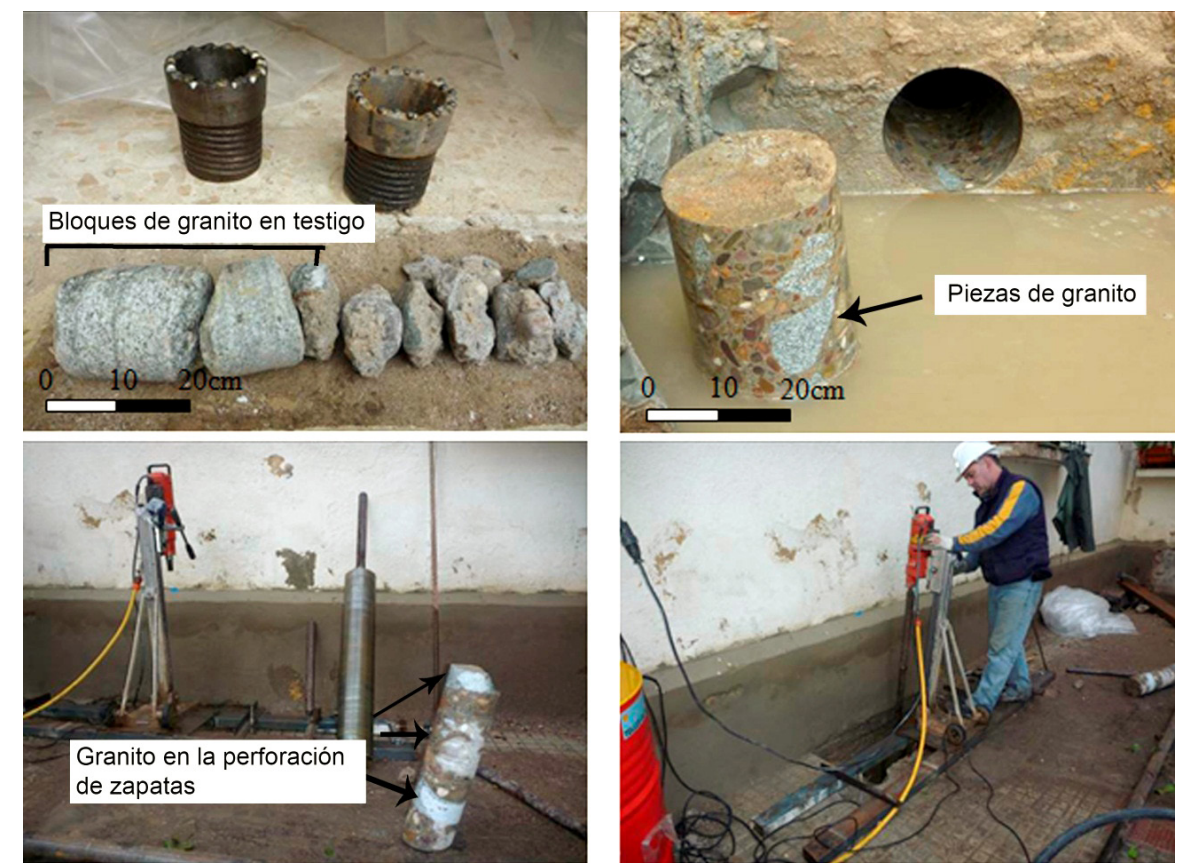

Figura 7. Perforación de las zapatas. Testigos (15 cm diámetro) extraídos donde se aprecia el uso de granito en la cimentación y equipo para su perforación.

rían a incumplir los compromisos adquiridos con los propietarios de las viviendas. La presencia de grandes piezas de granito en la cimentación (Figura 7), la cual se desconocía que fuese ciclópea al no detectarse en la caracterización estructural, generó la necesidad de buscar soluciones alternativas a la perforación de las zapatas, puesto que, con los rendimientos obtenidos en las primeras perforaciones, la ejecución de un micropilote podía requerir una jornada entera.

El rendimiento, definido al inicio de la obra por la empresa adjudicataria, para los micropilotes realizados en el exterior de la edificación y ejecutados por una máquina de perforación de elevadas prestaciones debía ser de $60 \mathrm{~m} /$ día, pero sólo se alcanzó un rendimiento situado entre 20 y $30 \mathrm{~m} /$ día. Para solventar este contratiempo se decidió realizar la perforación de las zapatas con coronas de diamante con brazo extensor, para abarcar el espesor completo de la zapata.

Estas circunstancias se vieron agravadas en el interior de las viviendas, donde el reducido gálibo requería la utilización de maquinaria de escasas dimensiones (y la distancia al punto de perforación hizo inviable el uso de la solución anterior).

Tras analizar otras posibilidades, se decidió utilizar una broca perforadora que, a la vez que gira, es alimentada por un compresor neumático que suministraba a través del interior de la vaina la energía necesaria para realizar la percusión de avance. Seguidamente, se procedió a realizar el micropilote a través de la perforación, mejorando considerablemente el rendimiento de la obra.

Se decidió realizar una prueba controlada y observar el comportamiento de paramentos y fisuras en el entorno. El rendimiento obtenido resultó satisfactorio y se pudieron completar en una jornada cinco perforaciones de zapatas. No se detectó aumento en las fisuras del edifico u otros síntomas que indicasen que la estructura estaba siendo afectada. Exteriormente el edificio ya se encontraba completamente recal- zado, favoreciendo la estabilidad del conjunto y evitando el aumento de asientos o movimientos.

El cambio del sistema de perforación de zapatas permitió recuperar el rendimiento planificado, 232 unidades de micropilotes de 27 metros de longitud fueron ejecutados en 94 días laborales, alcanzando un rendimiento global de $67 \mathrm{~m} /$ día, superior al teórico global de 60 m/día.

\section{COSTES DEL RECALCE}

A la hora de acometer la valoración económica de procesos y procedimientos constructivos de gran complejidad, como es el caso que nos atañe, los profesionales se enfrentan con multitud de cuestiones en la toma de decisiones, lo cual hace necesario recurrir a instrumentos adecuados que les permitan llevar a cabo esta labor de una manera eficaz, ordenada y con garantías (19). Para ello cuentan con los sistemas de clasificación de la información de construcción (SCIC) tales como: MasterFormat, Uniformat II, CESMM, CI / SFB incorporado al estándar ISO, Uniclass y OmniClass (19). Las bases de costes de la construcción en España poseen sus propios SCIC, y su mayor ámbito de implantación suele ser su entorno geográfico (20). Debido al entorno geográfico donde se centra el presente estudio de caso, el sistema de clasificación empleado es la Base de Costes de la Construcción de Andalucía (en adelante BCCA) por ser referente para las obras de la administración pública en la región (21). Es un sistema de ordenación y clasificación de las unidades de obra, flexible y adaptable, que permite cambios de gran importancia como han sido la incorporación de la gestión de residuos (20).

Por las connotaciones especiales que ofrecen las unidades de obras de recuperación, se ha optado por precios complejos o funcionales que recoge unidades constructivas completas con sus procedimientos de ejecución y actividades simultáneas representadas en precios unitarios simples. En la Tabla 1 se muestra el nuevo precio funcional de recuperación de zapata 
Tabla 1. Precio Unitario Funcional del recalce de zapata (PUF). Precios unitarios simples descompuestos que constituyen el PUF del recalce.

\begin{tabular}{|c|c|c|c|c|c|}
\hline \multicolumn{6}{|c|}{ Precio Unitario Funcional del recalce de zapata (PUF) } \\
\hline $\mathbf{0 3}$ & \multicolumn{5}{|c|}{ CIMENTACIONES } \\
\hline $\mathbf{O 3 R}$ & \multicolumn{5}{|c|}{ RECUPERACIONES } \\
\hline o3RZ & \multicolumn{5}{|c|}{ ZAPATAS } \\
\hline o3RZQoooo1 & $\mathbf{u}$ & \multicolumn{4}{|c|}{ RECALCE DE ZAPATA CORRIDA DE HORMIGÓN CICLÓPEO POR MICROPILOTAJE } \\
\hline \multicolumn{6}{|c|}{$\begin{array}{l}\text { Recuperación de cimentación mediante recalce por micropilotaje de zapara corrida de hormigón ciclópeo, incluso traba- } \\
\text { jos previos, descabezado, limpieza y doblado de armaduras. }\end{array}$} \\
\hline \multicolumn{6}{|c|}{ Medida la cantidad ejecutada. } \\
\hline Código & \multicolumn{2}{|r|}{ Concepto } & Cantidad & Precio & Importe (€) \\
\hline o3RZCoooo1 & $\mathrm{m}^{3}$ & Apertura de caja en acerado & 42,00 & 59,41 & $2.495,03$ \\
\hline o3RZRoooo1 & $\mathrm{u}$ & Perforación en zapata desde el exterior & 42,00 & 84,30 & $3 \cdot 540,60$ \\
\hline 03RZRoooo2 & $\mathrm{u}$ & Perforación en zapata desde el interior & 188,00 & 75,52 & $14.197,76$ \\
\hline o3RZKoooo1 & $\mathrm{m}$ & $\begin{array}{l}\text { Micropilote in situ exterior terrenos consist.baja } \\
\text { c/cam.acero Ø 60mm }\end{array}$ & 1092,00 & 59,68 & $65.170,56$ \\
\hline 03RZKoooo2 & $\mathrm{m}$ & $\begin{array}{l}\text { Micropilote in situ interior terrenos consist. } \\
\text { Baja c c/cam.acero Ø 6omm }\end{array}$ & 4587,20 & 66,43 & $304.727,70$ \\
\hline & & & \multicolumn{2}{|c|}{ Total Costes Directos } & $390.131,65$ \\
\hline
\end{tabular}

Precios unitarios simples descompuestos que constituyen el Precio Unitario Funcional del recalce de zapata (PUF).

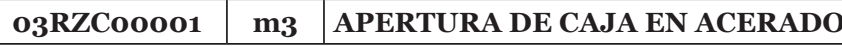

Apertura de caja en acerado, realizada con medios manuales hasta una profundidad máxima de 1,50 m, incluso perfilado de bordes, carga con medios manuales y traslado de residuos a cuba en obra, a una distancia máxima de $500 \mathrm{~m}$.

Medida en perfil natural

\begin{tabular}{|c|c|l|l|l|l|}
\hline Código & Ud & Concepto & Cantidad & Precio & Importe (E) \\
\hline TPoo100 & $\mathrm{h}$ & Peón especial & 3,100 & 18,28 & 56,67 \\
\hline MKоозоO & $\mathrm{h}$ & Carretilla mecánica basculante & 0,750 & 3,65 & 2,74 \\
\hline \multicolumn{7}{|l}{} & Costes Directos & 59,41 \\
\hline
\end{tabular}

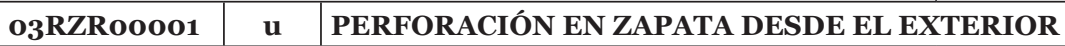

Perforaciones en zapata desde el exterior para micropilotaje, realizada mediante equipo de perforación por rotación con corona de diamante en la totalidad de la altura de la zapata, incluso retirada de testigo.

\begin{tabular}{|c|c|c|c|c|c|}
\hline \multicolumn{6}{|c|}{ Medida la cantidad ejecutada } \\
\hline Código & Ud & Concepto & Cantidad & Precio & Importe (€) \\
\hline TO02200 & $\mathrm{h}$ & Oficial $2^{\mathrm{a}}$ & 1,00 & 18,74 & 18,74 \\
\hline TPoo100 & $\mathrm{h}$ & Peón especial & 1,50 & 18,28 & 27,42 \\
\hline MP80001 & $\mathrm{h}$ & Equipo perforación c/corona diamante & 1,00 & 38,14 & 38,14 \\
\hline \multicolumn{5}{|c|}{ Total. Costes Directos } & 84,30 \\
\hline o3RZKoоoо1 & $\mathbf{m}$ & \multicolumn{4}{|c|}{$\begin{array}{l}\text { MICROPILOTE IN SITU EXTERIOR TERRENOS CONSISTENCIA BAJA C/CAMISA ACERO } \\
\emptyset 60 \mathrm{~mm}\end{array}$} \\
\hline
\end{tabular}

Micropilote in situ en exterior de edificio de diámetro exterior $140 \mathrm{~mm}$, armado con camisa de acero de $60,3 \mathrm{~mm}$ de $\varnothing$ interior y 5,5 $\mathrm{mm}$ de espesor de pared, hasta $27 \mathrm{~m}$ de profundidad, incluso descabezado, limpieza y doblado de armaduras; construido según EHE, NCSR-O2 y CTE.

\begin{tabular}{|c|c|c|c|c|c|}
\hline & & & & & \\
\hline Código & Ud & Concepto & Cantidad & Precio & Importe (€) \\
\hline MP80000 & $\mathrm{h}$ & Equipo perforación micropilotes & 0,183 & 29,36 & 5,37 \\
\hline MPoo6oo & $\mathrm{u}$ & Repercusión s/útil, transp. máquina & 1,000 & 1,82 & 1,82 \\
\hline GCoO200 & $\mathrm{t}$ & Cemento cem ii/a-l 42,5r a granel & 0,125 & 92,54 & 11,53 \\
\hline CA80080 & $\mathrm{m}$ & Camisa acero S $375 \mathrm{JR} \varnothing 60 \mathrm{~mm}$ & 1,000 & 26,46 & 26,46 \\
\hline TPoo100 & $\mathrm{h}$ & Peón especial & 0,590 & 18,28 & 10,79 \\
\hline TO02100 & $\mathrm{h}$ & Oficial $1^{\mathrm{a}}$ & 0,183 & 19,23 & 3,52 \\
\hline MCoo10o & $\mathrm{h}$ & Compresor dos martillos & 0,030 & 6,35 & 0,19 \\
\hline & & & Tota & tes Directos & 59,68 \\
\hline
\end{tabular}


03RZQ0ooo1, y los precios unitarios simples descompuestos creados: apertura de caja en acerado 03RZCoooo1, perforación en zapata desde el interior 03RZRoooo2 y micropilote in situ interior 03RZKooo02. Sólo se incluyen en la Tabla 1 los miropilotes exteriores, esto se debe a que los componentes de estos procesos por el interior son coincidentes, variando solo las cantidades.

La elaboración los nuevos precios funcionales de recuperación de zapata se ha realizado siguiendo la metodología utilizada en la BCCA y, a partir otros precios básicos existentes, modificando de manera independiente el sumando de mano de obra en el que se refleja el incremento que supone la diferencia de rendimiento. El importe de ejecución material (IEM) de la intervención en el edificio ascendió a 1.468.664,76 $€$ de los cuales 390.131,65 $€$ fueron el coste de recalce de la cimentación, representando el 26,56\%.

\section{CONCLUSIONES}

Las intervenciones en edificaciones en circunstancias de emergencia, y en concreto los recalces de cimentaciones se puede apoyar en tres posibles soluciones: incrementar su superficie de apoyo, mejorar la consistencia del terreno o profundizar la transmisión de carga a terreno competente. En el caso estudiado, un edificio de 40 viviendas en Sevilla, cercanas al Río Guadalquivir, afectado por la construcción contigua de un aparcamiento subterráneo, se adopta el método de miropilotes; debido, entre otras razones, a las condiciones del suelo.

Con el sistema establecido de recalce se consiguió el rendimiento planificado de 232 unidades de micropilotes de 27 metros de longitud ejecutados en 94 días laborales, alcanzan- do un rendimiento global de $67 \mathrm{~m} /$ día, superior al teórico de $54 \mathrm{~m} /$ día.

Conviene destacar que el entorno de emergencia social y técnica en el que se desarrollan este tipo de obras condiciona notablemente las soluciones constructivas a aplicar. El éxito de este tipo de intervenciones no radica en disponer de grandes presupuestos que permitan acometer complejas obras, más bien se compone de tres factores claves: realizar una correcta organización de la obra; en este caso de estudio se pudieron organizar los trabajos de las dos obras, la nuevas Facultades de Ciencias de la Salud de la Universidad de Sevilla y recuperación de edificio RENFE, establecer la consecución de los objetivos en un plazo temporal cercano y, por último, implicar a los propietarios en el proceso de ejecución de la obra a través de reuniones periódicas con la asociación de vecinos.

La aplicación de este procedimiento ha demostrado su efectividad y ha permitido conseguir los objetivos temporales marcados, dando cumplimiento a los compromisos adquiridos por la universidad con los propietarios de las viviendas. Con su divulgación se espera que la experiencia adquirida sea de utilidad a técnicos o gestores y sirva de referencia ante posibles situaciones similares futuras para que puedan, igualmente, gestionarse de manera positiva.

En trabajos futuros se evaluarán las otras actividades realizadas en la recuperación del edificio como cubiertas, revestimientos, urbanización, etc. que sirvan de referencia en intervenciones similares, desarrollando su correspondiente clasificación de los trabajos, sus rendimientos y costes. El caso particular estudiado servirá como base a un sistema general de clasificación sistemática de la construcción en trabajos de recuperación de edificios.

\section{REFERENCIAS}

(1) FEMA (2002). Rapid visual screening of buildings for potencial seismics hazards, a hand book. 2nd. Edition. Federal Emergency Management Agency. U.S. Dept. Of Homeland Security.

(2) Zhang, H., Xing, F. Liu, J. (2011). Rehabilitation decision-making for buildings in the Wenchuan area. Earthquake Construction Management and Economics. 29(6): 569-578, doi: http://dx.doi.org/10.1080/01446193.2011.569732.

(3) Ewing, R.C. (2011). Foundation repairs due to expansive soils: Eudora Welty House, Jackson, Mississippi. Journal of Performance of Constructed Facilities, 25(1): 50-55, doi: http://dx.doi.org/10.1061/(ASCE)CF.1943-5509.0000159.

(4) Wilder, D., Smith, G.C.G., Gómez, J. (2005). Issues in design and evaluation of Compaction Grouting for foundation repair. Geotechnical Special Publication, (130-142): 1739-1750.

(5) Seksinsky, E.J, Qubain, B.S. (1998). An underpinning solution to a persistent foundation settlement problem. Geotechnical Special Publication, (81): 202-2013.

(6) Lutenegger, A. J., Kemper, J.H.(2008). Preservation of historic structures using Screw-Pilefoundations. Structural Analysis of Historic Construction: Preserving Safety and Significance - Proceedings of the 6th International Conference on Structural Analysis of Historic Construction, SAHCo8, (2):1079-1086, doi: http://dx.doi.org/10.1201/9781439828229. ch123.

(7) Da Casa, F., Echevarría, E., Celis, F. (2012). El movimiento en la ejecución de recalces con inyección armada. Análisis de tres casos con movimientos previos. Informes de la Construcción, 64(528): 507-518, doi: http://dx.doi.org/10.3989/ ic.11.101.

(8) Da Casa, F., Echevarría, E., Celis, F. (2007). La intervención bajo rasante, la importancia de su conocimiento. La técnica de la inyección armada. Informes de la Construcción, 59(505): 21-35, doi: http://dx.doi.org/10.3989/ic.2007.v59. i505.498.

(9) Ministerio de Fomento. (1956). Expediente Administrativo 104+4o Viviendas en Plaza de Armas, Sevilla. Proyecto (SE-1415-VP) y expediente (SE-1518-VP). Archivo Central. Archivo histórico del Instituto Nacional de la Vivienda.

(10) AEN/CTN 103-GEOTECNIA. (2006). UNE-EN ISO 22476-3:2006 Investigación y ensayos geotécnicos. Ensayos de campo. Parte 3: Ensayo de penetración estándar. Asociación española de normalización (AENOR).

(11) Burland, J. B., Broms, B. B., De Mello, V.F. (1977). Behavior of Foundations and Structures, State of the art report. En 9th International Conference on Soil Mechanics and Foundation Engineering, (Vol. 2, pp. 495-546). Tokyo. 
(12) Da Casa, F., Echeverría, E., Celis, F. (2005). Tratamiento perimetral con inyecciones armadas para excavación para parking subterráneo en Sevilla. En Libro de actas de las $2^{a}$ Jornadas Luso- Espanholas de Geotecnia. Lisboa, Portugal: Ed. Sociedad Portuguesa de Geotecnia.

(13) Rodríguez-Mayorga, E., Yanes, E., Compan, V., Saez-Perez, A. (2013). La restauración del templo parroquial de San Dionisio. La inyección como método de reparación de estructuras de fábrica. Informes de la Construcción, 65(529): 5-16, doi: http://dx.doi.org/10.3989/ic.11.130.

(14) Erdemgil, M., Sağlam, S., Bakır, B.S. (2007, 05-07 de diciembre). Utilization of highly expansive polymer injection to mitigate seismic foundation for existing structures. En 8th Pacific Conference on Earthquake Eng. Singapore.

(15) Dei Svaldi, A., Favaretti, M., Pasquetto, A., Vinco, G. (2005, 18-19 de julio). Analytical modelling of the soil improvement by injections of high expansion pressure resin. En Congress proceedings of 6th International Conference on Ground Improvement Techniques (pp. 577-584). Coimbra.

(16) Buzzi, O., Fityus, S., Sasaki, Y., Sloan, S. (2008). Structure and properties of expanding polyurethane foam in the context of foundation remediation in expansive soil. Mechanics of Materials, 40(12): 1012-1021, doi: http://dx.doi. org/10.1016/j.mechmat.2008.07.002.

(17) AIN. (1968). UNI 6350-68 Materias plásticas celulares rígidas - Determinación de las características a la compresión. Asociación italiana de normalización.

(18) Foti, S., Manassero, M. (2009, 18-19 de noviembre). Rinforzo e adeguamento delle fondazioni per sollecitazioni statiche e dinamiche. En XXII ciclo de las Conferencias de Geotécnica. Turín, Italia.

(19) Marrero, M., Ramirez-de-Arellano, A. (2010). The building cost system in Andalusia: application to construction and demolition waste management. Construction Management and Economics, 28(5): 495-507, doi: http://dx.doi. org/10.1080/01446191003735500.

(20) Marrero, M., Solis-Guzman, J., Molero Alonso, B., Osuna-Rodriguez, M., Ramirez-de-Arellano, A. (2011). Demolition Waste Management in Spanish Legislation. The Open Construction \& Building Technology Journal, 5: 162-173, doi: http://dx.doi.org/10.2174/1874836801105010162.

(21) BCCA, S. L. (2013). Memoria Base de Costes de la Construcción de Andalucía. Sevilla. http://www.juntadeandalucia.es/ fomentoyvivienda/portal-web/web/texto/b28c72fc-4818-11e2-804b-e3c39o5d6ca6. 